\title{
L'impact du déboisement sur l'azote minéral susceptible d'être absorbé par un taillis de chêne vert (Quercus ilex $L$ ) en reconstitution
}

\author{
A Merzouki *, P Lossaint + , G Billes, M Rapp \\ CEPE/CNRS, Centre d'écologie fonctionnelle et évolutive \\ BP 5051, Route de Mende, Montpellier, France
}

(Reçu le 17 avril 1990; accepté le 8 octobre 1990)

\begin{abstract}
Résumé - L'étude de l'effet du déboisement pratiqué dans les taillis peu productís de chêne vert (Quercus ilex $L$ ) du Sud de la France sur l'activité biologique des sols rouges méditerranéens a été poursuivie de janvier 1984, date d'une coupe à blanc, jusqu'en avril 1986. Un aspect important de l'étude a consisté à estimer la dynamique des quantités d'azote potentiellement assimilables par la végétation aussi bien sous un taillis témoin de 42 ans que dans un site en reconstitution où les rejets des souches sont âgés de 1 à 2 ans. L'étude a montré que durant une année la disponibilité en azote, pour les rejets de 1 à 2 ans d'âge, est essentiellement nitrique, alors qu'elle est mixte (ammoniacale et nitrique) pour le taillis âgé de 42 ans. Cette disponibilité serait de 47,4 à $118,6 \mathrm{~kg}^{\circ} \mathrm{ha}^{-1} \cdot \mathrm{an}^{-1}$ pour les rejets de souches, constituée uniquement de nitrates et de 25,6 à $63,9 \mathrm{~kg}^{\circ} \mathrm{ha}^{-1} \cdot \mathrm{an}^{-1}$ pour le taillis témoin, où l'ammonium représente environ $43 \%$ de l'azote minéral disponible.
\end{abstract}

déboisement / disponibilité en azote / nitrate / rejets de souches / taillis de chêne vert / Quercus Ilex L

Summary - The impact of deforestation on the mineral nitrogen available during restoration of the holm oak (Quercus ilex L) coppice. The impact of deforestation on the biological activity of a mediterranean red soil was studied from January 1984 to April 1986 within a holm oak (Quercus ilex L) stand after clearcut. Part of the study involved the estimation of the amount of nitrogen available for vegetation both in the mature holm oak coppice and in the 1-2-yr-old recovery stand. The study indicates that the new shoots essentially took up nitrate nitrogen; however, the mature site used both nitrate and ammonium nitrogen as nitrogen source. The availability of mineral nitrogen,

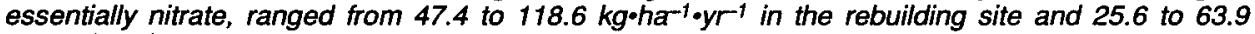
$\mathrm{kg}^{\circ} \mathrm{ha}^{-1} \cdot \mathrm{yr}^{-1}$ in the mature stand. At the control site, ammonium nitrogen represented $43 \%$ of the total nitrogen available.

deforestation / nitrogen availability / nitrate / shoots / holm oak coppice / Quercus ilex L

* Correspondance et tirés à part. 


\section{INTRODUCTION}

Dans la région méditerranéenne, l'écosystème taillis de chêne vert (Quercus ilex $L$ ) a été périodiquement exploité par des coupes avec des rotations de 25 à 30 ans. Ces déboisements ont des conséquences multiples sur la dynamique de cet écosystème. En effet l'exportation du matériel végétal modifie aussi bien les conditions microclimatiques, le pédoclimat, le stock de matière organique du sol, le compartiment biomasse microbienne (Merzouki et Lossaint, 1990; Merzouki et al, 1989a, b), que la dynamique des nutriments et leur répartition au sein de la végétation (Ed-Derfoufi, 1986).

Dans cet ecosystème méditerranéen, l'azote et l'eau constituent les éléments limitants du développement et de la reconstitution du peuplement soumis à la coupe. En effet, la production primaire dépend, d'une part de la quantité et de la forme de l'azote minéral disponible dans le sol (exclusivement $\mathrm{NH}_{4}^{+}$ou $\mathrm{NO}_{3}^{-}$ou $\mathrm{NH}_{4}^{+}$et $\mathrm{NO}_{3}^{-}$à la fois) et d'autre part de la capacité d'assimilation de cet élément par la végétation à différents stades de développement.

L'absorption azotée a fait l'objet de nombreuses études, notamment par Kirkby (1981), Novoa et Loomis (1981), Mengel et Kirkby (1982), Nadelhoffer et Aber (1984) et Salsac et Chaillou (1984). Plusieurs auteurs estiment que les espèces forestières seraient plutôt ammoniacophiles, surtout dans les écosystèmes tempérés (Bormann et Likens, 1979; Cole, 1981; Reiners, 1981). Dans les sols de ces formations la nitrification est lente et l'ammonium reste la forme azotée dominante, disponible pour la végétation. D'autres, tels Robertson et Vitousek (1981) ou Roberston (1982) ont montré que beaucoup de ces écosystèmes ont une nitrification potentielle très élevée. Ellenberg (1977), quant à lui, a classé les écosystèmes forestiers selon l'aptitude de leurs sols à fournir aux plantes, soit du $\mathrm{NH}_{4}^{+}$, soit du $\mathrm{NO}_{3}^{-}$, soit les deux à la fois.

Le présent travail se propose d'étudier dans un peuplement de chêne vert les changements qui ont affecté la disponibilité en azote et sa nature (ammoniacale, nitrique ou mixte) chez des rejets de souches de 1 à 2 ans d'âge, puis de quantifier cette disponibilité potentielle et de la comparer entre un taillis âgé et un peuplement en voie de reconstitution.

\section{MATÉRIEL ET MÉTHODES}

La station étudiée fait partie de la forêt domaniale de Puéchabon (2 000 ha); elle est située à $25 \mathrm{~km}$ au Nord-Ouest de Montpellier, à une altitude de $250 \mathrm{~m}$ (latitude $43^{\circ} 44^{\prime} 30^{\prime \prime} \mathrm{N}$, longitude $3^{\circ} 35^{\prime} 50^{\prime \prime}$ E). Agé de 40 ans au moment de la coupe et constitué essentiellement de chênes verts de 3 à $5 \mathrm{~m}$ de hauteur, ce peuplement est considéré comme représentatif des taillis de chêne vert de la zone méditerranéenne française (Floret et al, 1989).

Le climat est de type méditerranéen subhumide avec une pluviosité moyenne de 1000 $\mathrm{mm}$ (Debussche et Escarre, 1983) très variable d'une année à l'autre, $1078 \mathrm{~mm}$ en 1984 et 535 $\mathrm{mm}$ en 1985. La température moyenne mensuelle est de $2{ }^{\circ} \mathrm{C}$ en janvier et de $23^{\circ} \mathrm{C}$ en juillet.

Le sol est de type rouge fersiallitique à réserve calcique, sur roche mère calcaire dure du Jurassique. If a une profondeur variable et existe le plus souvent sous la forme de poches dans les fissures du karst. L'abondance des cailloux varie de 50 à $80 \%$ du volume du profil. L'horizon organique ne dépasse pas $5 \mathrm{~cm}$.

Dans le cadre d'une étude des conséquences du déboisement sur le fonctionnement du système taillis de chêne vert et sur la reconstitution de la végétation, la totalité des arbres sur une surface d'environ $2500 \mathrm{~m}^{2}$ a été coupée entre 5 et $10 \mathrm{~cm}$ au dessus du sol, puis débitée sur place avant d'être évacuée. Pour la comparaison entre le taillis et la coupe, nous avons pris d'une part cette situation et d'autre part une situation dans le taillis lui-même. 


\section{Mineralisation de l'azote}

Dans les deux sites étudiés (sous forêt et site déboisé), 6 échantillons de sol sont prélevés dans l'horizon supérieur entre 0 et $15 \mathrm{~cm}$, débarrassés de la litière, des racines et des cailloux par tamisage à $4 \mathrm{~mm}$. Le sol homogénéisé est réparti en 2 lots; l'un servira pour l'incubation sur le terrain et l'autre sera transporté au laboratoire pour déterminer les teneurs résiduelles en azote minéral.

\section{Incubation et dosage}

Sur le terrain, le sol tamisé et homogénéisé est réparti dans des boîtes métalliques de $830 \mathrm{ml}$, perforées latéralement et à la base. Six boîtes ont été placées dans l'horizon correspondant aux prélèvements $(0-15 \mathrm{~cm})$ pendant 30 jours. Le protocole des incubations est représenté par le schéma des périodes d'incubation sur le terrain. Le sol était ainsi incubé dans des conditions de température et d'humidité proches de celles du milieu.

L'azote minéral du sol a été extrait par une solution de sulfate de potassium normal $(1 \mathrm{~N})$, le rapport extractant/sol étant de 5/1. Le mélange est agité durant $1 \mathrm{~h}$, centrifugé à 4000 tours/ min pendant $15 \mathrm{~min}$ puis filtré. Pour le dosage de $\mathrm{NH}_{4}^{+}$, l'extrait a été additionné d'H $\mathrm{SO}_{4}$ concentré ( $36 \mathrm{~N}$ ) à raison de $2 \%$.

Pour le dosage de $\mathrm{NO}_{3}^{-}$l'extrait est récupéré dans une solution de sulfate de cuivre acidifiée à raison de $10 \%$.

L'ammonium et le nitrate ont été dosés colorimétriquement.

\section{Calcul de l'azote minéral mis à la disponibilité de la végétation}

A partir de la production mensuelle d'azote minéral in situ (équation 1), on peut estimer la quantité potentiellement utilisable par la végétation à partir de l'horizon $0-15 \mathrm{~cm}$ durant chaque période d'incubation. En effet l'essentiel du système radiculaire et de l'activité biologique sont localisés à ce niveau. Cette absorption potentielle est calculée selon l'équation 2 , utilisée no- tamment par Nadelhoffer et Aber (1984) et Djellali et al (1985).

L'azote minéral sous quelque forme qu'il soit est exprimé en ppm. Et dans le souci d'exprimer la production annuelle d'azote minéral dans cet

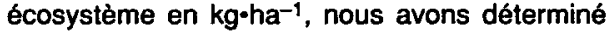
que le pourcentage des cailloux dans thorizon 0-15 cm varie de 50 à $80 \%$ du volume. Soit une quantité de sol biologiquement active qui serait de $435 \mathrm{~T} \cdot h a^{-1}$ à $1087 \mathrm{~T} \cdot \mathrm{ha}^{-1}$.

Les périodes d'incubation sur le terrain sont :

$$
\begin{aligned}
& \mathrm{N}_{\mathrm{i}}(t)-\cdots \mathrm{N}(t+1) \\
& N_{\mathrm{i}}(t+1)-\cdots \mathrm{N}(t+2) \\
& N_{i}(t+2)----N(t+3) \\
& \mathrm{N}_{\mathrm{i}}(t+3)--\mathrm{N}(t+4) \\
& N_{i}(t+4)
\end{aligned}
$$

$\mathrm{N}_{\mathrm{i}}(t)$ : azote minéral initial de la première période d'incubation

$N(t+1)$ : azote minéral après 30 jours d'incubation sur le terrain

$N_{\mathrm{i}}(t+1)$ : azote minéral résiduel ou initial de la $2^{\circ}$ période.

On en déduit :

$\mathrm{N}(t+1)-\mathrm{N}_{\mathrm{i}}(t)=\mathrm{N}$ produit après un mois d'incubation in situ

$\mathbf{N}$ produit $-\mathbf{N}_{i}(t+1)=\mathbf{N}$ disponible pour la végétation

En effet : $N$ résiduel $=N$ minéralisé $-(N$ absorbé par la végétation + $N$ réorganisé par la microflore $+\mathbf{N}$ perdu par lessivage, etc) et $\mathbf{N}$ produit in situ $=\mathbf{N}$ minéralisé $-\mathbf{N}$ réorganisé par la microflore

Donc : $\mathbf{N}$ produit in situ $-\mathbf{N}$ résiduel $=\mathbf{N}$ absorbé par les plantes + N perdu

On appelle quantité azoté potentiellement disponible (NPD), la quantité d'azote absorbée par la végétation plus celle qui est perdue (lessivage, volatilisation, etc).

En résumé :

$$
\begin{aligned}
& \mathrm{N}-\mathrm{NH}_{4}^{+} \mathrm{PD}=\mathrm{N}-\mathrm{NH}_{4}^{+}(t+1)-\mathrm{N}-\mathrm{NH}_{4}^{+} \mathrm{i}(t+1) \\
& \mathrm{N}-\mathrm{NO}_{3}^{-} \mathrm{PD}=\mathrm{N}-\mathrm{NO}_{3}^{-}(t+1)-\mathrm{N}-\mathrm{NO}_{3}^{-} \mathrm{i}(t+1) \\
& \mathrm{N}-\min \mathrm{PD}=\mathrm{N}-\mathrm{NH}_{4}^{+} \mathrm{PD}+\mathrm{N}-\mathrm{NO}_{3}^{-} \mathrm{PD}
\end{aligned}
$$

\section{Analyse statistique des résultats}

L'analyse de la différence entre les deux situations étudiées - des quantités d'azote mis à la 
disponibilité de la végétation - est estimée à l'aide du test de Student au seuil de $P=0,05$ et $P=0,01$.

\section{RÉSULTATS}

La dynamique de l'azote du sol a été suivie de mars 1984 à avril 1986 (Merzouki et al, 1989b). Dans ce travail, on n'a considéré que les données expérimentales de la période allant de janvier à décembre 1985, soit une année. Par contre, les corrélations entre les teneurs résiduelles en ammonium et les teneurs issues de l'incubation in situ sont établies à partir de 16 mesures sous forêt et 14 mesures dans le site déboisé. Certaines teneurs, considérées comme aberrantes, ont été écartées : elles correspondent soit à des périodes de fortes pluies qui ont entraîné un engorgement des boîtes d'incubation, soit à des accidents sur le terrain qui ont abouti au déterrement et à une dessiccation du sol des boîtes.

\section{Production d'azote minéral}

Les variations mensuelles de la production d'azote minéral in situ ont été estimées dans l'horizon 0-15 cm aussi bien sous couvert forestier que dans le site déboisé. Cette production (tableau I) varie de 27,9 à $69,8 \mathrm{~kg} \cdot \mathrm{ha}^{-1} \cdot \mathrm{an}^{-1}$ sous forêt et de 41,0 à $102,4 \mathrm{~kg} \cdot \mathrm{ha}^{-1} \cdot \mathrm{an}^{-1}$ dans le site déboisé. L'azote minéral est uniquement sous forme nitrique dans le site déboisé. Dans le site forestier la part du nitrate varie de 15,7 a $39,2 \mathrm{~kg} \mathrm{ha}^{-1} \cdot \mathrm{an}^{-1}$.

\section{L'azote minéral disponible pour la végétation}

La dynamique et la disponibilité potentielle d'azote pour la végétation durant l'année 1985 a été estimée mensuellement. Les teneurs sont présentées dans le tableau II. II en ressort que cette disponibilité de l'azote est positive 6 mois par an dans le taillis âgé alors qu'elle l'est de 9 mois par an dans le peuplement en reconstitution.

Dans le taillis de chêne vert de 42 ans l'azote minéral disponible dans la solution du sol est mixte, elle est à base de $\mathrm{N}$ $\mathrm{NH}_{4}{ }^{+}$et $\mathrm{N}-\mathrm{NO}_{3}-$. Les teneurs de $\mathrm{N}$ minéral total susceptible d'être mensuellement consommé varient de $-1,8$ à 16,7 ppm, soit des quantités annuelles qui varient de 25,6 à $63,9 \mathrm{~kg}^{\circ} \mathrm{ha}^{-1} \cdot \mathrm{an}^{-1}$. La part du nitrate varie mensuellement de $-1,8$ à $12,3 \mathrm{ppm}$, soit, 14,6 à $36,4 \mathrm{~kg} \mathrm{ha}^{-1} \cdot \mathrm{an}^{-1}$ ce qui correspond à $57 \%$ de l'azote minéral total disponible pour la végétation (tableau III). Dans le site déboisé où les rejets de souches sont âgés de 1 à 2 ans, l'azote minéral disponible est plutôt sous forme nitrique. L'azote minéral susceptible d'être absorbé par les souches et les rejets varie de $-8,0$ à 26,1 ppm par mois, soit entre 39,0 et $97,4 \mathrm{~kg} \cdot \mathrm{ha}^{-1}$ par année.

Le nitrate constitue la totalité de l'azote minéral disponible pour la végétation en reconstitution. Mensuellement, les teneurs

Tableau I. Production de $\mathrm{N}-\mathrm{NH}_{4}{ }^{+}, \mathrm{N}-\mathrm{NO}_{3}{ }^{-}$et $\mathrm{N}$ minéral total sous forêt et dans le site déboisé, exprimée en $\mathrm{kg} \cdot \mathrm{ha}^{-1} \cdot \mathrm{an}^{-1}$ par rapport à la fourchette de terre fine de l'horizon $0-15 \mathrm{~cm}$.

\begin{tabular}{lccc}
\hline & $\mathrm{N}-\mathrm{NH}_{4}{ }^{+}$ & $\mathrm{N}-\mathrm{NO}_{3}^{-}$ & $\mathrm{N}$ minéral total \\
\hline Forêt & $12,2-30,6$ & $15,7-39,2$ & $27,9-69,8$ \\
Coupe & $-9,1-22,9$ & $50,2-125,3$ & $41,0-102,4$ \\
\hline
\end{tabular}




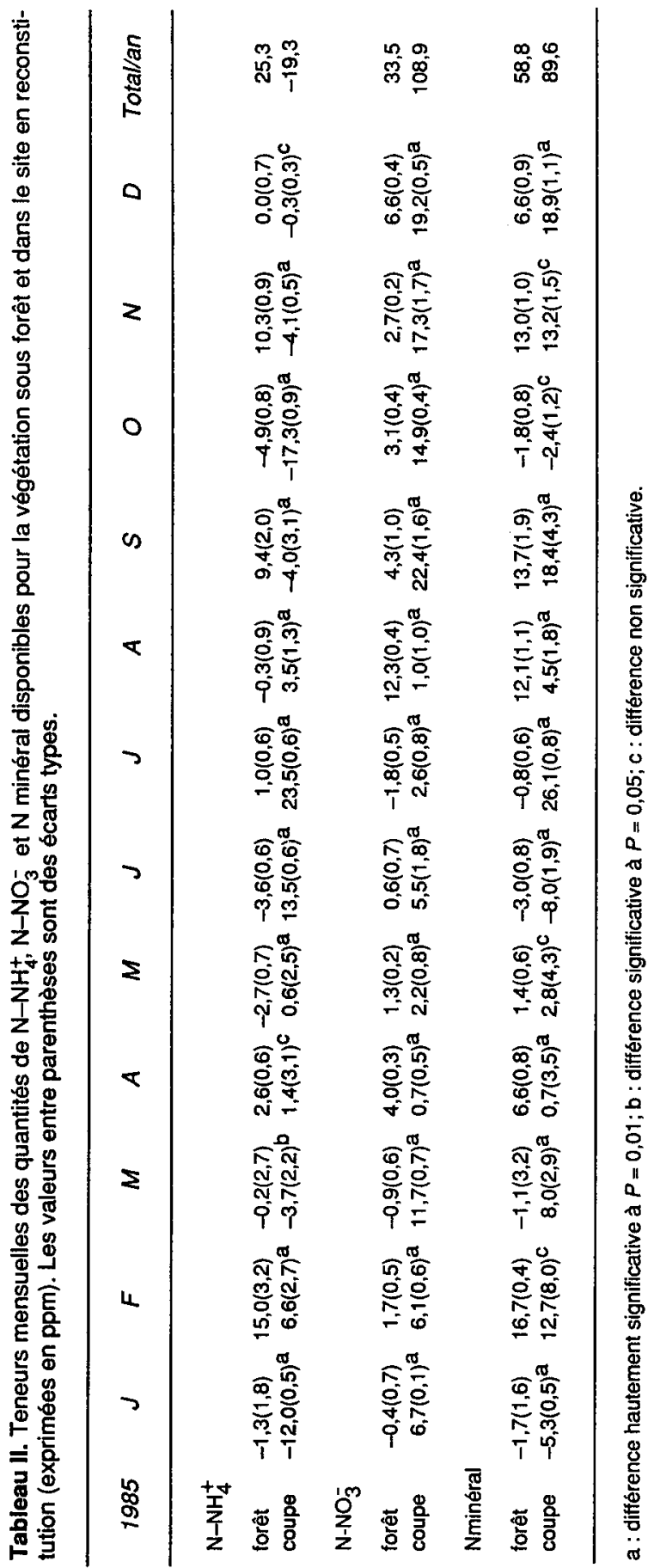


Tableau III. Quantités deN- $\mathrm{NH}_{4}{ }^{+}, \mathrm{N}-\mathrm{NO}_{3}{ }^{-}$et $\mathrm{N}$ minéral total disponible pour la végétation sous forêt et dans le site en reconstitution, exprimées en $\mathrm{kg} \cdot \mathrm{ha}-1 \cdot \mathrm{an}-1$ par rapport à la fourchette de terre fine de l'horizon $0-15 \mathrm{~cm}$

\begin{tabular}{lccccc}
\hline & $\mathrm{N}-\mathrm{NH}_{4}{ }^{+}$ & $\mathrm{N}-\mathrm{NO}_{3}^{-}$ & $\mathrm{N}$ minéral total & $\%{\mathrm{~N}-\mathrm{NH}_{4}{ }^{+}}^{-}$ & $\% \mathrm{NO}_{3}^{-}$ \\
\hline Forêt & $11,0-27,5$ & $14,6-36,4$ & $25,6-63,9$ & 43,0 & 57,0 \\
Coupe & $-8,4--21,0$ & $47,4-118,4$ & $39,0-97,4$ & 0 & 100,0 \\
\hline
\end{tabular}

en nitrate sont de 0,7 à $22,4 \mathrm{ppm}$, soit en quantité 47,4 à $118,4 \mathrm{~kg} \cdot \mathrm{ha}^{-1} \cdot \mathrm{an}^{-1}$, ce qui correspond à $100 \%$ de l'azote minéral total.

\section{DISCUSSION}

Dans les écosystèmes forestiers, l'absorption d'azote par la végétation (herbacée, arbustive et arborée) constitue avec l'immobilisation microbienne, le processus de fixation de l'azote le plus important (Vitousek et Matson, 1984). Dans le sol, l'azote est mieux protégé sous forme d'ammonium, ce cation pouvant être fixé. Cette partie du cycle de l'azote est peu explorée. En effet, l'ammonium non échangeable constitue jusqu'à $25 \%$ de l'azote total du sol (Nommik, 1981; Nommik et Vahtras, 1982); il constitue une réserve potentielle pour la végétation.

Cole (1981) estime que l'anion nitrate a peu d'importance dans les écosystèmes stables, le rapport ammonium/nitrate serait de 10/1. Cet anion très mobile est absorbé par la végétation ou lessivé; il n'entre que faiblement dans les processus d'immobilisation/minéralisation. Les microorganismes hétérotrophes préfèreraient l'ammonium (Jones et Richards, 1977).

Dans l'écosystème taillis en reconstitution, les rejets de souches âgés de 1 à 2 ans se développent dans un sol riche en nitrate. Les nouvelles conditions stationnelles (pédoclimat, matière organique du sol) et la nature du sol stimulent l'activité des nitrificateurs (Merzouki et al, 1989b; Merzouki et Lossaint, 1990). Les teneurs d'ammonium résiduelles et issues de l'incubation in situ (en absence d'absorption radiculaire et de lessivage) (fig 1) montrent qu'il existe une étroite relation entre ces deux aspects de l'ammonium aussi bien sous forêt où $r=0,80^{\star \star}$ que dans le site

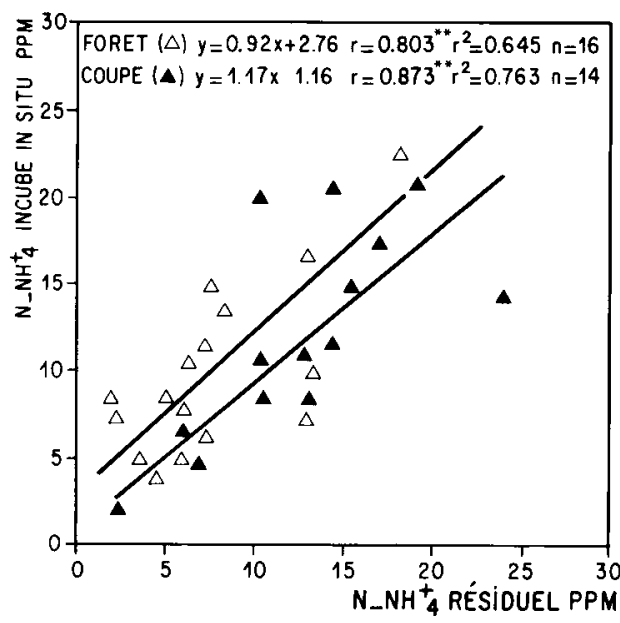

Fig 1. Relation entre les teneurs résiduelles de $\mathrm{N}-\mathrm{NH}_{4}{ }^{+}$et les teneurs de $\mathrm{N}-\mathrm{NH}_{4}{ }^{+}$issues de l'incubation in situ sous forêt et dans le site débojsé. 
déboisé où $r=0,87^{\star \star}$. Les variations de concentration de ce cation seraient donc le résultat de l'activité des microorganismes nitrificateurs qui entrent en compétition avec la végétation. Nadelhoffer et Aber (1984) ont observé que les quantités d'azote minéral issu du sol incubé sur le terrain sont équivalentes à celles du sol environnant (non incubé).

Dans le site déboisé, la disponibilité en azote est en majorité sous forme de nitrate. La nutrition azotée des rejets est donc essentiellement à base de cet anion (tableau III).

Bowen (1981) conclut que l'absorption de cet anion est maximale lorsque les teneurs dans la solution du sol sont élevées. Une activité nitrate-réductase dans le système radiculaire favoriserait aussi cette absorption (Adams et Attiwill, 1982). Bien que la transformation du nitrate en ammoniaque, lors de l'assimilation de l'azote minéral, soit un processus biologiquement coûteux, cette absorption est un avantage dans les écosystèmes ou l'élément azoté est un facteur limitant; elle réduit les pertes par lessivage (Ho et Trappe, 1980) et augmente la productivité primaire.

Ainsi la nutrition azotée, énergiquement la plus coûteuse, aboutit à la productivité primaire la plus élevée (Salsac et Chaillou, 1984). Dans le taillis de chêne vert en reconstitution, Ed-Derfoufi (1986) a noté que l'incrément mensuel de biomasse des jeunes pousses durant la saison de végétation est en moyenne de $101,5 \mathrm{~kg} \cdot \mathrm{ha}^{-1}$ et de $76,0 \mathrm{~kg}^{\circ} \mathrm{ha}^{-1}$ dans le taillis âgé. L'auteur a conclu que la coupe a donné «un coup de jeunesse" aux souches.

En étudiant la dynamique des nutriments dans les différents compartiments de la végétation (bois, tronc et pousses, feuilles de 1 an, feuilles de 2 ans et litière) du taillis adulte et des rejets de souches, Ed Derfoufi (1986) a estimé que dans le site déboisé, l'absorption azotée calculée à partir de la formule (absorption = immobilisation + restitution - transfert interne) est significativement corrélée à la production ( $r$ $\left.=0,88^{* *}\right)$. La quantité d'azote absorbée est de $55,4 \mathrm{~kg}^{\circ} \mathrm{ha}^{-1} \cdot \mathrm{an}^{-1}$, ce qui correspond aux valeurs de l'azote disponible calculé à partir de la minéralisation nette in situ.

Plusieurs plantes vasculaires utilisent les deux formes d'azote minéral (Driessche Van Der, 1978; Ho et Trappe, 1980). Il semble que ce soit le cas du peuplement âgé du chêne vert. La quantité d'azote absorbée durant une année est composée de $43 \%$ d'ammonium et de $57 \%$ de nitrate. Dans le site déboisé le nitrate constitue $100 \%$ de l'azote minéral total. Dans le cas où l'azote est essentiellement sous forme ammoniacale, les espèces végétales s'adaptent à la situation en investissant dans un système radiculaire très dense. En effet ce cation est très peu mobile et son transfert à la surface radiculaire se fait par diffusion. Une abondance du système racinaire, couplée par la présence de mycorhize serait bénéfique pour la nutrition ammoniacale (Bledsoe et Zasoski, 1983).

Le chêne vert adulte absorbe à la fois $\mathrm{NH}_{4}{ }^{+}$et $\mathrm{NO}_{3}$. Lorsque le nitrate est présent en grande quantité, par exemple à la suite de l'activité des nitrificateurs consécutive aux changements pédoclimatiques et trophiques induits par la coupe, cette espèce privilégie l'absorption sous forme de nitrate. Ceci permet également d'éviter les pertes de nitrate par lessivage à la suite de la mise à nu du sol.

Le chêne vert et les espèces forestières seraient donc "opportunistes" en s'adaptant et en utilisant l'élément azoté le plus abondant dans la solution du sol. Ceci nous permet d'avancer l'idée qu'il est difficile de classer des écosystèmes forestiers selon l'aptitude de leurs sols à fournir l'ammonium, le nitrate ou les deux à la fois pour la nutrition azotée. 


\section{CONCLUSION}

L'azote minéral susceptible d'être absorbé par le taillis en reconstitution est uniquement sous forme de nitrate et varie de 47,4 a $118,4 \mathrm{~kg}^{\prime} \mathrm{ha}^{-1} \cdot \mathrm{an}^{-1}$. Dans le taillis adulte, l'azote minéral susceptible d'être absorbé varie de 25,6 à $63,9 \mathrm{~kg} \mathrm{ha}^{-1} \cdot \mathrm{an}^{-1}$ avec environ $43 \%$ d'ammonium et $57 \%$ de nitrate.

\section{REMERCIEMENTS}

L'auteur tient à remercier F Ed-Derfoufi et $F$ Romane pour leurs conseils lors de l'élaboration de ce texte.

\section{RÉFÉRENCES}

Adams MA, Attiwill PM (1982) Nitrogen mineralization and nitrate reduction in suests. Soil Boil Biochem 14, 197-202

Bledsoe CS, Zasoski RJ (1983) Effects of ammonium and nitrate on growth and nitrogen uptake by mycorrhizal Douglas fir seedlings. Plant Soil 71, 445-454

Bowen GD (1981) Approaches to nutritional physiology. In Productivity in perpetuity. Proceedings of the Australien forest nutrition Workshop Canberra Australia, 79-91

Bormann FH, Likens GE (1979) Pattern and process in forested ecosystem. Springer Verlag, New York, $253 p$

Cole DW (1981) Nitrogen uptake and translocation by forest ecosystems. In: Terrestrial nitrogen cycles (Clark FE and Rosswall T, eds) Ecol Bull 33, 219-231

Debussche M, Escarre J (1983) Cartes des isohyètes inter-annuelles dans le Montpelliérais. Document du CEPE, CNRS, Montpellier

Djellali $N$, Billes $G$, Bounaga $N$, Lossaint $P$ (1985) Étude de l'activité biologique des sols de la steppe à alfa d'Algérie. Minéralisation du carbone et de l'azote. Acta Oecol Oecol Plant 6, 289-307

Driessche Van Der R (1978) Response of Douglas fir seedlings to nitrate and ammonium nitrogen sources at different levels of $\mathrm{pH}$ and iron supply. Plant Soil 49, 607-623

Ed-Derfoufi $F$ (1986) Gestion et dynamique des nutrients dans un écosystème forestier de chêne vert. Thèse, Montpellier, 130 p

Ellenberg $H$ (1977) Stickstoff als Standorstsfaktor, insbesondere für mitteleuropäische Pflanzengesellschaften. Oecol Plant 12, 1-12

Floret C, Galan MJ, Le Floc'H E, Rapp M, Romane $F$, Blanchard A, Grandjanny $M$, Maistre $M$, Perret $P$ (1989) Organisation de la structure, de la biomasse et de la minéralomasse d'un taillis ouvert de chêne vert (Quercus ilex L). Acta Oecol Oecol Plant 10, 245-263

Ho I, Trappe JM (1980) Nitrate reductase activity of nonmycorrhizal Douglas fir rootlets and some associated mycorrhizal fungi. Plant Soil $54,395-398$

Jones JM, Richards BN (1977) Effect of reforestation on turnover of $\mathrm{N}$-labelled nitrate and ammonium in relation to changes in soil microflora. Soil Biol Biochem 9, 383-392

Kirkby EA (1981) Plant growth in relation to nitrogen supply. In: Terrestrial nitrogen cycles (Clark FE and Rosswall T, eds) Ecol Bull 33, 249-267

Mengel K, Kirkby EA (1982) Principle of plant nutrition. Switzerland International Potash Institue, $655 \mathrm{p}$

Merzouki A, Lossaint P, Rapp M (1989a) L'effet d'une coupe à blanc sur le compartiment biomasse microbienne d'un sol rouge méditerranéen. Pedobiologia 33, 221-228

Merzouki A, Lossaint P, Rapp $M$, Billes $G$ (1989b) L'effet d'une coupe à blanc sur la minéralisation de l'azote d'un sol rouge méditerranéen. Rev Écol Biol Sol 26, 133-154

Merzouki A, Lossaint P (1990) L'impact du déboisement sur le compartiment «matière organique" d'un sol rouge méditerranéen. Bull Ecu': -ous presse)

Nadelhoffer KJ, Aber JD (1984) Seasonal patterns of amonium and nitrate uptake in nine temperate forest systems. Plant Soil 80, 321335

Nommik H (1981) Fixation and biological availability of ammonium on soil clay minerals. In: Terrestrial nitrogen cycles (Clark FE and Roswall T, eds) Ecol Bull 33, 273-280 
Nommik H, Vahtras K (1982) Retention and fixation of ammonium and ammonia in soils. In: Nitrogen in agricultural soils (Stexensen FJ, ed) Agronomy 22, 123-166

Novoa R, Loomis RS (1981) Nitrogen and plant production. Plant Soil 58, 177-204

Reiners WA (1981) Nitrogen cycle in relation to ecosystem succession. In: Terrestrial nitrogen cycles (Clark FE, Rosswall T, eds). Bull 33, 507-528

Robertson GP (1982) Factors regulating nitrification in primary and secondary succession. Ecology 63, 1033-1040
Robertson GP, Vitousek PM (1981) Nitrification potentials in primary and secondary succession. Ecology 63, 1561-1573

Salsac L, Chaillou S (1984) Nutrition azotée des végétaux, importance physiologique et écologique de la fourniture d'azote sous forme nitrique ou ammoniacale. Bull Soc Écophysiol $9,111-128$

Vitousek PM, Matson PA (1984) Mechanisms of nitrogen retention in forest ecosystems: a field experiment. Science 225, 51-52 